\title{
Algoritmo para la provisión de QoS en redes inalámbricas 802.11 basado en restricciones de retardo extremo a extremo
}

\section{Algorithm for the QoS Provision in Wireless Networks 802.11 Based on End-to-End Delay Restrictions}

\author{
EVELIO ASTAIZA-HOYOS \\ Magister en ingeniería electrónica y telecomunicaciones \\ Universidad del Quindío \\ eastaiza@uniquindio.edu.co \\ Armenia, Colombia \\ HÉCTOR FABIO BERMÚDEZ-OROZCO \\ Magister en ingeniería electrónica y telecomunicaciones \\ Universidad del Quindío \\ hfbermudez@uniquindio.edu.co \\ Armenia , Colombia
}

LUIS FREDDY MUÑOZ-SANABRIA

Magister en computación

Fundación Universitaria de Popayán

lfreddyms@fup.edu.co

Popayán , Colombia

Fecha de recibido: 22/01/2015

Fecha de aceptado: 10/07/2015

Forma de citar/How to cite: ASTAIZA, Evelio; BERMÚDEZ, Héctor y MUÑOZ, Luis. Algoritmo para la provisión de QoS en redes inalámbricas 802.11 basado en restricciones de retardo extremo a extremo. Rev. UIS Ingenierías, 2015, vol. 14, no. 2, p.p. 57-63.

DOI: http://dx.doi.org/10.18273/revuin.v14n2-2015006 (c) (1) $\Theta$

\section{RESUMEN}

Actualmente en el mundo de las telecomunicaciones existe el problema de garantizar que los servicios de usuario puedan ser transportados adecuadamente en redes inalámbricas. Para dichas redes se debe certificar que el máximo retardo extremo a extremo experimentado se encuentre por debajo de los umbrales establecidos, con los cuales se pueda garantizar una calidad de servicio (QoS) necesaria sobre todo en aplicaciones y servicios que requieren transferencia de información en tiempo real, como lo son las llamadas de voz, videollamadas, juegos en línea, entre otros. Por lo tanto, es necesario que los retardos sean mínimos para llevar a cabo la correcta comunicación. En el presente artículo, se aborda el problema desde la perspectiva de la teoría de juegos no cooperativos utilizando argumentos de equilibrio como lo es el equilibrio de satisfacción (ES) ya que este concepto de solución garantiza cumplir con las restricciones de retardo extremo a extremo proporcionando la QoS requerida en este aspecto.

PALABRAS CLAVE: Calidad del servicio, Equilibrio de satisfacción, Juegos no cooperativos, Redes inalámbricas 802.11, Retardo extremo a extremo, Teoría de juegos. 


\section{ABSTRACT}

Currently in the world of telecommunication there is the problem to ensure that user services can be transported properly in wireless networks, such networks must certify that the maximum end-to-end delay experienced is below the thresholds necessary to provide quality of service (QoS) especially in applications and services that require transfer of information in real time, such as voice calls, video calls, online games, among others; therefore, it is necessary that the delays are minimal to perform correct communication. In this paper, the problem is approached from the perspective of the theory of noncooperative games using equilibrium arguments such as the equilibrium of satisfaction (ES) as this solution concept ensures meet delay constraints end to end providing the required QoS in this regard.

KEYWORDS: Quality of Service, Satisfaction Equilibrium, Noncooperative Games, 802.11 Wireless Networks, End-to-End Delay, Game Theory.

\section{INTRODUCCIÓN}

Las comunicaciones inalámbricas son indispensables para el desarrollo de las actividades cotidianas, ya que el acceso a la red es necesario para la transmisión de datos provenientes de servicios de texto, audio y vídeo, entre otras. Por esta razón, se han desarrollado las diferentes aplicaciones que permiten la comunicación entre los diferentes usuarios. Esta transmisión de datos en algunos casos se debe realizar en tiempo real, ya que servicios como audio y video presentan algunos requerimientos sobre los recursos asignados en la red para su adecuado funcionamiento, tales como el ancho de banda, relación señal a ruido, retardo extremos a extremo, entre otros. Por lo anterior, esta investigación trabaja en el diseño de un algoritmo que garantice que el máximo retardo extremo a extremo sufrido se encuentra por debajo de los umbrales establecidos por la Unión Internacional de Telecomunicaciones - UIT (ITU-T, 1998).

El entorno de desarrollo del algoritmo está conformado por dos usuarios y dos puntos de acceso (AP), este modelo puede ser extendido a un escenario $\mathrm{n}^{*} \mathrm{n}$, es decir múltiples usuarios y múltiples AP. Para dar solución al problema se hace uso de la teoría de juegos, herramienta matemática que permite examinar el comportamiento estratégico de los participantes, que actúan motivados por la maximización de sus utilidades y suponen que los otros participantes son racionales (Vela Lindon, 2011).

Para el desarrollo de esta investigación, inicialmente se analiza el argumento de equilibrio de Nash. El equilibrio de Nash se define como un estado de la red en la que los usuarios (jugadores) no pueden mejorar su calidad de servicio por cambiar unilateralmente el Access Point al cual están realizando la conexión; el adjetivo "unilateral" hace referencia al carácter no cooperativo de las elecciones individuales. Es posible que en un equilibrio de Nash la situación se puede mejorar para todos por medio de un cambio simultáneo de estrategia por parte de varios ju- gadores (Sánchez, 2009). Por otro lado, el equilibrio de satisfacción (ES) representa cualquier estado de la red donde los usuarios satisfacen sus requerimientos. En esta medida, el equilibrio de satisfacción (ES) es el que garantiza cumplir con las restricciones de retardo extremo a extremo asociado a QoS y es por eso que se escoge en la solución presentada.

El objetivo del presente artículo es proponer un algoritmo basado en restricciones de retardo extremo a extremo en el nivel físico y/o enlace, que permita proporcionar QoS en redes 802.11 bajo presunciones de mapeo adecuado de dichos requerimientos de tiempo desde los niveles superiores.

\section{TRABAJOS RELACIONADOS}

Mediante el uso de la teoría de juegos se han resuelto diferentes problemas que se han presentado en las comunicaciones inalámbricas, dando como resultado soluciones eficientes, tales como: la asignación de recursos, la formación de redes, el enrutamiento, la gestión de interferencias, la gestión de redes dinámicas, la asignación de espectros, la transmisión cooperativa, la radio cognitiva, la seguridad, las redes ad hoc y la instalación de agentes inalámbricos (Dimitri et al., 2014).

En (Khodaian Khalaj, 2010), (Hou Kumar, 2010) y (Meshkati et al., 2009) se ha aplicado un marco de maximización de utilidad con el fin de optimizar el rendimiento de redes multi-hop de acceso aleatorio, sin embargo el retardo no es limitado y se proponen algoritmos para retardos de transmisión, se estudia el problema de la maximización de la utilidad para los clientes con requisitos de QoS basado en retardo en redes inalámbricas y es un trabajo en el que se aborda los pagos o ganancias de energía-retardo en las redes inalámbricas, se estudian utilizando un marco de teoría de juegos, una red de clase y de acceso múltiple, se considera que los usuarios eligen sus potencias de transmisión y posiblemente, las tasas 
de transmisión de manera distribuida para maximizar sus propias utilidades, al tiempo que satisface sus requisitos de retardo y calidad de servicio (QoS) respectivamente.

En (Meshkati et al., 2009) se propone un modelo basado en teoría de juegos para estudiar el problema uniendo potencia y control de la frecuencia con restricciones de calidad de servicio en las redes de acceso múltiple. En el juego propuesto, cada usuario busca para elegir su potencia de transmisión y frecuencia de una manera distribuida y egoísta con el fin de maximizar su propia utilidad y al mismo tiempo satisfacer sus requisitos de QoS.

En la Universidad del Quindío se han desarrollado dos proyectos relacionados con esta investigación, en (Trujillo, 2012) se desarrolla un algoritmo para la selección de Access Point desde la teoría de juegos bajo restricciones de potencia, utilizando métodos de solución como equilibrio de Nash, equilibrio de satisfacción y equilibrio de satisfacción eficiente; obteniendo en este último trabajo mejores resultados, ya que en este se adecuan las condiciones de los valores mínimos de capacidad y potencia que le permiten satisfacer la capacidad umbral requerida por cada usuario para garantizar QoS. En (Londoño, 2013) se plantea la selección de Access Point en redes 802.11 basado en restricciones de ancho de banda utilizando teoría de juegos no cooperativos donde se considera garantizar calidad de servicio en el nivel físico a los usuarios desde la perspectiva exclusiva de la capacidad mínima requerida para cada servicio, bajo presunciones de mapeo adecuado de las tasas de transferencia de información desde los niveles superiores.

\section{DESARROLLO}

\subsection{Equilibrio de Nash y Equilibrio de Satisfacción}

Entre las soluciones mediante los argumentos de equilibrio se encuentra el equilibrio de (Nash, 1950) y el equilibrio de satisfacción (Ross Chaib-Draa, 2006). El modelo de juego que se plantea es no cooperativo. En este modelo cada jugador toma una posición egoísta buscando maximizar sus utilidades mediante las estrategias que tenga para jugar y se modela como juego estático en el que los jugadores toman las decisiones simultáneamente.

El juego se modela como (Nash, 1950):

$$
G=\left\{K,\left\{S_{k}\right\}_{k \in K},\left\{u_{k}\right\}_{k \in K}\right\}
$$

en donde $K$ es el número de jugadores o usuarios de la red. Para todo $k \in K$, el conjunto $S_{k}$ representa el conjunto de acciones del usuario $k$ y la función $u_{k}: S \rightarrow R$ es la función de utilidad del jugador $k$.
Un equilibrio de Nash es un conjunto de estrategias (una para cada usuario) que constituyen la mejor respuesta que cada uno puede dar a las acciones de los otros usuarios. En un equilibrio de Nash el usuario maximiza su utilidad esperada, tomando las acciones de los otros como dadas. De esta manera ningún jugador se siente tentado a modificar su estrategia (Nash, 1950).

\section{Proposición 1 (Existencia del equilibrio de Nash - EN):}

Sea el juego $G=\left\{K,\left\{S_{k}\right\}_{k \in K},\left\{u_{k}\right\}_{k \in K}\right\}$ tal que, para todo jugador $k$, se cumple:

1. $s_{k}$ es un subconjunto no vacío, compacto y convexo de un espacio $R^{k}$.

2. $u_{k}$ es continua en todo su dominio $S=S_{1} \times S_{2} \times \ldots \times$ $S_{k}$ y es cuasicóncava en la variable $S_{k}$ entonces, existe al menos un EN en estrategias puras de $G$ (Pérez et al., 2004).

\section{Proposición 2 (Existencia del equilibrio de Nash - EN:)}

En todo juego finito $G=\left\{K,\left\{S_{k}\right\}_{k \in K},\left\{u_{k}\right\}_{k \in K}\right\}$ existe al menos un EN en estrategias mixtas (Astaiza, 2013).

En la proposición 2 se afirma que, al menos en los juegos finitos, siempre existe como mínimo un equilibrio de Nash (en estrategias puras o mixtas).

Se establece una condición suficiente para la existencia del EN, dado que $G$ es finito, pero que no es condición necesaria. De este modo aplicando únicamente este teorema se comprueba la existencia del equilibrio de Nash para el modelo de juego planteado debido a que es un juego finito con número de jugadores y estrategias finitas.

Entre estos conceptos de solución también se encuentra el equilibrio de satisfacción, este radica en la importancia de tener satisfechos a los jugadores teniendo en cuenta las mínimas necesidades requeridas por cada uno de ellos en cuanto a las utilidades. Bajo la perspectiva del juego no cooperativo, se busca que cada usuario elija una estrategia que tenga relacionado el costo más bajo pero que finalmente el jugador alcance el nivel de satisfacción deseado, es decir, bajo este escenario, este no está interesado en desviarse de la estrategia seleccionada. En este criterio se puede utilizar un concepto que representa de una u otra manera la satisfacción de un jugador con las 
utilidades a partir de los límites establecidos mediante la función de utilidad. En el modelo de juego cada usuario elige una estrategia, cada una de las cuales representa el rendimiento promedio en el tiempo que cada jugador tendrá para ejecutar las diferentes aplicaciones multimedia y a su vez satisfacen las restricciones de retardo extremo a extremo.

Proposición 3 (Definición del equilibrio de Satisfacción - ES:)

Un perfil de acciones $s^{+}$es un ES para el juego

$$
G=\left\{K,\left\{S_{k}\right\}_{k \in K},\left\{u_{k}\right\}_{k \in K},\left\{f_{k}\right\}_{k \in K}\right\}
$$

si $\forall k \in K, s_{k}{ }^{+} \in f_{-k}\left(s_{k}{ }^{+}\right)$, donde $f_{-k}\left(s_{-k}{ }^{+}\right)$está definida como

$$
f_{-k}\left(s_{-k}^{+}\right)=\left\{s_{k} \in S_{k}: u_{k}\left(s_{k}, s_{-k}\right) \geq \Gamma_{k}\right\}
$$

Donde $\Gamma_{k}$ representa las mínimas restricciones de retardo extremo a extremo para cada usuario $k$ (Perlaza et al.).

Entonces,

$$
S_{E S} \triangleq\left\{s \in S: \forall k \in K, s_{k} \in f_{k}\left(s_{-k}\right)\right\} \subseteq S
$$

Proposición 4 (Existencia del equilibrio de Satisfacción - ES:)

La existencia de un ES en el juego $G=\left\{K,\left\{S_{k}\right\}_{k \in K}\right.$, $\left.\left\{u_{k}\right\}_{k \in K},\left\{f_{k}\right\}_{k \in K}\right\}$ depende principalmente de los umbrales determinados por la función de utilidad. Por ejemplo, sea la correspondencia $F: S \rightarrow 2^{S}$ la cual se define como $F(s)=\left(f_{1}\left(s_{-k}\right), f_{2}\left(s_{-k}\right), . ., f_{K}\left(s_{-k}\right)\right)$, por lo tanto un equilibrio de satisfacción existe si y solo si:

$$
\exists \mathbf{s} \in \mathbf{S}: \mathbf{s} \in F(\mathbf{s})
$$

Esta formulación permite utilizar los teoremas existentes de punto fijo para proporcionar condiciones necesarias y suficientes para la existencia del equilibrio de satisfacción. Por ejemplo, del teorema de punto fijo de Kakutani puede escribirse la siguiente proposición (Perlaza et al.).

\section{Proposición 5 (Existencia del Equilibrio de Satisfac- ción:)}

En el juego $G=\left\{K,\left\{S_{k}\right\}_{k \in K},\left\{u_{k}\right\}_{k \in K},\left\{f_{k}\right\}_{k \in K}\right\}$, sea $S$ el conjunto de acciones no vacío, compacto y convexo, y sea la correspondencia $F(\mathbf{s})$ la cual presenta un grafo cerrado, no vacío y convexo sobre el conjunto de acciones $S$, luego el juego $\mathrm{G}$ tiene al menos un ES.

En la referencia (Perlaza et al.), se determina la existencia de al menos un equilibrio de satisfacción eficiente ESE.

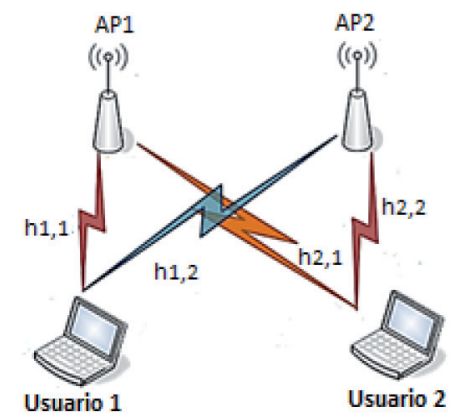

Figura 1. Sistema de comunicaciones en un escenario con 2 AP y 2 usuarios.

\subsection{Modelo del Sistema}

Para esta investigación se plantea un modelo asociado a una red inalámbrica, con los elementos necesarios para llevar a cabo el desarrollo del proyecto y encontrar los equilibrios y soluciones ante los problemas de retardo de extremo a extremo.

El sistema se plantea como un juego no cooperativo en el cual los jugadores toman decisiones para su beneficio personal, lo cual no impide que en algunos casos dicha toma de decisiones pueda favorecerlos a todos, como es lo que se busca en los juegos cooperativos, adicionalmente con un escenario estático donde los participantes toman sus decisiones simultáneamente. El sistema se conforma por dos usuarios y dos AP que acceden a internet en una zona WiFi como se muestra en la Figura 1.

El juego se denota de la forma:

$$
G=\left\{(i=1,2,3, \ldots, I) ; y_{0, k}, y_{1, k}, y_{2, k}, \ldots, y_{I, k} ; u_{k}\left(y_{k}, y_{-k}\right)\right\}
$$

en donde $i$ es el número de jugadores o usuarios de la red, $y_{1, k}$ es el rendimiento promedio en el tiempo. Esta variable cumple con las restricciones de retardo extremo a extremo las cuales equivalen a cada una de las posibles estrategias que tiene disponible cada jugador y $u_{k}$ como función de utilidad que dependerá básicamente del rendimiento promedio en el tiempo, de acuerdo con la estrategia seleccionada por cada jugador.

En el artículo "Delay-Based Network Utility Maximization"(Neely, 2013) se trabaja con una función de utilidad que cumple con los requerimientos para la ejecución de esta investigación, en donde $g(y)$ es una función continua y cóncava del Vector L-dimensional $y(t)=\left(y_{1}, y_{L}\right)$, se utiliza $y$ para representar el rendimiento promedio en el tiempo en cada enlace. 
La función puede tomar valores positivos o negativos, y está definido para $0=y=1$.

Mientras $v_{l}$ es una constante que limita la tasa de cambio de la función de utilidad, con respecto al rendimiento de cada enlace en la red, esta constante $v_{l}$ toma valores mayores a cero y puede ser igual para todos los enlaces con el fin de garantizar equidad proporcional (Neely, 2013).

$$
g(y)=\log \left(1+v_{l} * y_{l}\right)
$$

La función $g(y)$ se utiliza para encontrar la solución bajo los argumentos de equilibrio planteados anteriormente, teniendo en cuenta el desempeño de las redes IEEE 802.11 y las características de este estándar para satisfacer los requerimientos necesarios.

En (Neely, 2013) se trabaja con tiempo discreto con intervalos de time slots normalizados $t=0,1,2$, Si hay $L$ enlaces, los paquetes llegan al azar a cada slot y se ponen en cola por separado para la transmisión sobre cada enlace, $A_{l}(t)$ es el número de paquetes que llegan a un enlace en un slot, se representa por $D(\tau)=\left(D_{\tau}(\tau), D_{L}(\tau)\right)$ el vector de descarte para el slot $\tau$, dado el vector $L$ dimensional $y(t)=\left(y_{1}, y_{L}\right)$, donde $y$ se utiliza para representar el rendimiento promedio en el tiempo en cada enlace. $\lambda_{l}=E\left[A_{l}(t)\right]$ es una tasa de llegada para el enlace $l, Z_{L}(t)$ representa las colas virtuales para cada enlace y $T_{l}(t)$ es el tiempo entre llegada de paquetes.

\subsection{Metodología para la solución del juego}

El significado de la palabra solución hace referencia a la decisión óptima, la que más le conviene al agente que se plantea el problema. Además cuando hay varias soluciones, todas ellas son igualmente deseables para el agente. En términos intuitivos se llama solución de un juego a un conjunto de perfiles de estrategias tal que es razonable pensar que los jugadores tomarán decisiones pertenecientes a dicho conjunto, y se llama concepto de solución de un juego a un procedimiento que permita obtener, de manera precisa y bien argumentada, una solución (Pérez et al., 2004).

Se obtiene la matriz de utilidades, la cual es una herramienta de análisis de la teoría de juegos que permite interpretar las utilidades de cada usuario cuando realiza la selección de un Access Point. En la Tabla 1 se encuentra la matriz de utilidades para el modelo del sistema planteado en la Figura 1, se encuentra las posibles estrategias para acceder al punto de acceso que cumpla con los requisitos de cada uno de los usuarios.
Cuadro 1. Matriz de utilidades en el juego 2 × 2 en canal interferente.

\begin{tabular}{|c|c|c|c|}
\hline Usuario & \multicolumn{3}{|c|}{ US 2 } \\
\hline \multirow{3}{*}{ US 1 } & AP & AP1 & AP2 \\
\cline { 2 - 4 } & AP1 & $g_{11}=\log \left(1+v_{l} y_{11}\right)$ & $g_{11}=\log \left(1+v_{l} y_{11}\right)$ \\
& & $g_{21}=\log \left(1+v_{l} y_{21}\right)$ & $g_{22}=\log \left(1+v_{l} y_{22}\right)$ \\
\cline { 2 - 4 } & AP2 & $g_{12}=\log \left(1+v_{l} y_{12}\right)$ & $g_{12}=\log \left(1+v_{l} y_{12}\right)$ \\
& & $g_{21}=\log \left(1+v_{l} y_{21}\right)$ & $g_{22}=\log \left(1+v_{l} y_{22}\right)$ \\
\hline
\end{tabular}

Las condiciones para cada mejor respuesta son:

$B R_{1}\left(A P_{1}\right)= \begin{cases}A P 1 & \text { si } \log \left(1+v_{l} y_{11}\right) \geq \log \left(1+v_{l} y_{12}\right) \\ A P 2 & \text { si } \log \left(1+v_{l} y_{12}\right) \geq \log \left(1+v_{l} y_{11}\right)\end{cases}$

$B R_{1}\left(A P_{2}\right)= \begin{cases}A P 1 & \text { si } \log \left(1+v_{l} y_{11}\right) \geq \log \left(1+v_{l} y_{12}\right) \\ A P 2 & \text { si } \log \left(1+v_{l} y_{12}\right) \geq \log \left(1+v_{l} y_{11}\right)\end{cases}$

$B R_{2}\left(A P_{1}\right)= \begin{cases}A P 1 & \text { si } \log \left(1+v_{l} y_{21}\right) \geq \log \left(1+v_{l} y_{22}\right) \\ A P 2 & \text { si } \log \left(1+v_{l} y_{22}\right) \geq \log \left(1+v_{l} y_{21}\right)\end{cases}$

$B R_{2}\left(A P_{2}\right)= \begin{cases}A P 1 & \text { si } \log \left(1+v_{l} y_{21}\right) \geq \log \left(1+v_{l} y_{22}\right) \\ A P 2 & \text { si } \log \left(1+v_{l} y_{22}\right) \geq \log \left(1+v_{l} y_{21}\right)\end{cases}$

Las 4 posibles situaciones que se pueden presentar en el juego de acuerdo a las estrategias seleccionadas son:

1. $\left(A P_{1}, A P_{1}\right)$ cuando $\log \left(1+v_{l} y_{11}\right) \geq \log \left(1+v_{l} y_{12}\right)$ y $\log \left(1+v_{l} y_{21}\right) \geq \log \left(1+v_{l} y_{22}\right)$

2. $\left(A P_{1}, A P_{2}\right)$ cuando $\log \left(1+v_{l} y_{11}\right) \geq \log \left(1+v_{l} y_{12}\right)$ y $\log \left(1+v_{l} y_{22}\right) \geq \log \left(1+v_{l} y_{21}\right)$

3. $\left(A P_{2}, A P_{1}\right)$ cuando $\log \left(1+v_{l} y_{12}\right) \geq \log \left(1+v_{l} y_{11}\right)$ y $\log \left(1+v_{l} y_{21}\right) \geq \log \left(1+v_{l} y_{22}\right)$

4. $\left(A P_{2}, A P_{2}\right)$ cuando $\log \left(1+v_{l} y_{12}\right) \geq \log \left(1+v_{l} y_{11}\right)$ y $\log \left(1+v_{l} y_{22}\right) \geq \log \left(1+v_{l} y_{21}\right)$

En el Algoritmo1, se presenta la propuesta de algoritmo que permite encontrar los equilibrios de satisfacción, a partir de la selección de una estrategia y con ella la selección de un AP para cada usuario:

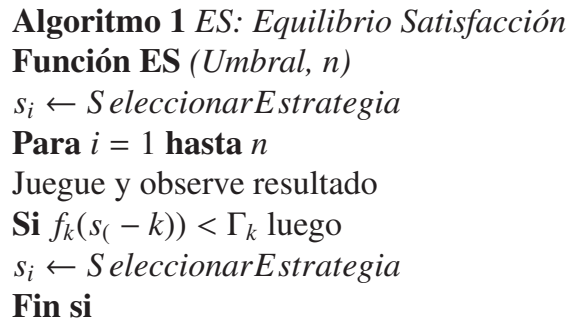

\section{Fin para Retornar $s_{i}$}

En el Algoritmo 1 se tiene: el contador $n$ el cual define el número de repeticiones del juego, la función Seleccionar Estrategia escoge aleatoria y uniformemente una 
estrategia del espacio de estrategias. En este caso siempre se estará buscando una estrategia cuando la utilidad sea menor al umbral requerido y de esta manera una vez todos los jugadores se encuentran satisfechos, no existirá agente alguno que desee cambiar de estrategia, dado que se ha alcanzado la condición de equilibrio.

\section{DISCUSIÓN Y RESULTADOS}

Para evaluar las estrategias de cada uno de los usuarios, se tienen en cuenta las restricciones de retardo de extremo a extremo establecidas por la ITU en la recomendación UIT-T G.114. Retardos inferiores a 100ms pueden afectar a tareas muy interactivas (por ejemplo, algunas aplicaciones vocales, de videoconferencia y de datos interactivos) (ITU-T, 2003).

Los retardos de extremo a extremo pueden ocurrir cuando los paquetes tomen un largo periodo en alcanzar su destino, debido a largas colas o tomen rutas menos directas para evitar congestión de la red. Para dar solución al problema planteado en este proyecto se aplican dos conceptos de solución y se desarrolla el algoritmo para alcanzar los resultados esperados. Cabe resaltar que en el Equilibrio de Satisfacción los usuarios están interesados en garantizar la QoS mínima.

Para realizar la simulación de la solución mediante el equilibrio de satisfacción fue necesario expresar los máximos retardos de extremo a extremo en términos del rendimiento, esto se realizó teniendo en cuenta algunos parámetros como el tamaño promedio de cada paquete es de 1500 bytes, el tamaño de time slot que es de $20 s$ para DSSS. Así entonces para el valor límite de retardo requerido por el servicio, y asumiendo que solo un paquete se envía en un time slot, se puede obtener el rendimiento asociado al retardo como la cantidad de bits que se enviaría en el tiempo de retardo, para posteriormente calcular la utilidad asociada a dicho retardo como lo ilustra la ecuación 7. Realizando este procedimiento se obtiene que para un retardo de $100 \mathrm{~ms}$ el rendimiento es del orden de 5.4Mbps y la utilidad asociada es aproximadamente 12. Con estos valores se implementa la simulación del algoritmo 1, en la cual se define un conjunto de 40 estrategias, cada una de las cuales representa el rendimiento de cada usuario en el enlace a cada uno de dos posibles AP. El umbral $\Gamma_{k}$ utilizado es de 12 de acuerdo al retardo de $100 \mathrm{~ms}$ manejados en la simulación. El proceso de selección de estrategias se realiza de manera secuencial y se calcula la utilidad alcanzada por cada usuario de acuerdo a la estrategia seleccionada y como se establece en la ecuación 7. De esta manera se obtiene la figura 2, donde se visualizan con líneas discontinuas de color azul

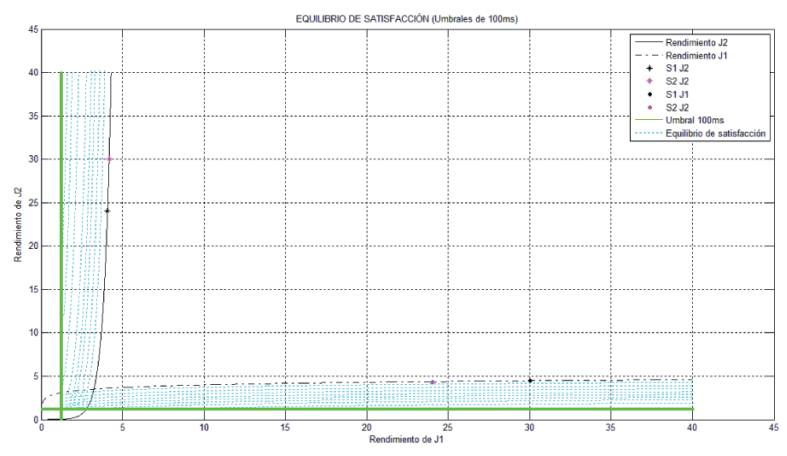

Figura 2. Región de satisfacción para el jugador 1 y jugador 2.

saturado los valores de la utilidad de las estrategias que permiten superar el umbral asociado al retardo manejado de $100 \mathrm{~ms}$. Para el cálculo de la utilidad en cada caso se toma el valor constante $v_{l}$ de manera arbitraria en 8 oportunidades con valores entre 10 y 70 para permitir la visualización de la región de equilibrio de satisfacción. De la misma forma, se traza en líneas negras continuas y discontinuas la utilidad asociada al valor $v_{l}=100$; así mismo se trazan los umbrales correspondientes en color verde.

Para efectos de simulación se asume que en el sistema actuarán diez usuarios, para visualizar bien el concepto de equilibrio de satisfacción, por lo tanto la tasa requerida asumida es diez veces mayor a la real.

En la Figura 2 se visualizan los resultados obtenidos para el concepto de solución basado en el equilibrio de satisfacción (ES), la sección que es de color azul saturado comprende la zona que cumple el equilibrio de satisfacción, es decir el lugar en donde se cumple con los requisitos de QoS y a su vez las restricciones de retardo establecida de $100 \mathrm{~ms}$, por lo cual cualquier punto dentro de esta región corresponde a un par de estrategias seleccionadas por los jugadores que les permite garantizar sus requerimientos de retardo extremos a extremo para la provisión de QoS.

\section{CONCLUSIONES}

El desarrollo de esta investigación permite destacar la importancia de la aplicación de la teoría de juegos en la innovación y resolución de problemas en el campo de las telecomunicaciones. A través de la teoría de juegos se da solución al problema planteado, satisfaciendo las restricciones de retardo extremo a extremo en las redes IEEE 802.11 . 
Mediante los argumentos de solución de equilibrio como el equilibrio de satisfacción se obtuvo el resultado esperado, ya que los dos usuarios acceden a los AP cumpliendo con las restricciones de retardo extremo a extremo que estable la UIT para servicios de tiempo real. En el caso de ES, utilizan los recursos necesarios para ejecutar las aplicaciones y cumplir con los requisitos de QoS.

El concepto de Equilibrio de Satisfacción es una herramienta poderosa que permite generar soluciones eficientes a problemas de asignación de recursos basados en restricciones, dado que a diferencia el equilibrio de Nash, cada jugador solo toma los recursos necesarios de red que son suficientes para satisfacer sus restricciones.

\section{TRABAJOS FUTUROS}

Con el desarrollo de esta investigación se resalta la aplicación de la teoría de juegos en la resolución de problemas que se presentan actualmente en el ámbito de las telecomunicaciones. Por esta razón es importante continuar con el proceso en donde el paso a seguir es el de unificar los proyectos realizados anteriormente (Trujillo, 2012) y (Londoño, 2013), para lo cual se planteará la solución del juego bajo estrategias de potencia y de ancho de banda. Adicionalmente se deben garantizar restricciones de retardo extremo a extremo para abarcar las problemáticas de la provisión de QoS en redes inalámbricas 802.11.

\section{REFERENCIAS}

Astaiza, E. Introducción a la teoría de juegos en comunicaciones inalámbricas. Trabajo de ascenso de categoría Universidad del Quindío Armenia, Quindío, 2013.

Dimitri, E.; Charilas; Athanasios, D.; Panagopoulos A survey on game theory applications in wireless networks. http://www.sciencedirect.com/science/article/pii/s1389128610002239, , 2014.

Hou, I.; Kumar, P. Utility maximization for delay constrained qos in wireless. in CA: IEEE, (pp. 1-9), 2010.

ITU-T SERIE G: Sistemas y medios de transmision, sistemas $y$ redes digitales conexiones y circuitos telefónicos internacionales.Características generales de los sistemas nacionales que forman parte de conexiones internacionales. G.114 Recommendation International Telecommunications Union, 1998.

ITU-T G.114 Recommendation. Technical Report International Telecommunications Union, 2003.

Khodaian, A.; Khalaj, B. Delay-constrained utility maximization in multi-hop random access networks. IET, Communications, 4, 19081918, 2010.

Londoño, A. Selección de Access Point en redes 802.11 basado en restricciones de ancho de banda. Trabajo de final de pregrado Universidad del Quindío, Programa de Ingeniería Electrónica, 2013.
Meshkati, F.; Poor, H.; Schwartz, S.; Balan, R. Energy-efficient resource allocation in wireless networks with quality of service constraints. Communications, IEEE Transactions on, 57, 3406-3414, 2009.

Nash, J. Equilibrium points in n-person games. Proceedings of the National Academy of Sciences of the United States of America, 50, 1950.

Neely, M. Delay-based network utility maximization. Networking, IEEE/ACM Transactions on, 21, 41-54, 2013.

Pérez, N.; Jimeno, P.; Cerda, T. Teoría de Juegos. Madrid, España: Pearson Education, 2004.

Perlaza, H., S.and Tembine; S., L.; Debbah, M. Satisfaction equilibrium: A general framework for qos provisioning in self-configuring networks,

Ross, S.; Chaib-Draa, B. Satisfaction equilibrium: Achieving cooperation in incomplete information games. En 19th Canadian Conf. on Artificial Intelligence (pp. 196-197). Quebec, 2006.

Sánchez, I. Teoría de Juegos volumen 1. Madrid, España: Kalamo libros, 2009.

Trujillo, D. Selección de Access Point en redes 802.11 garantizando mínima capacidad para QoS:una perspectiva desde la teoría de juegos no cooperativos. Trabajo de final de pregrado Universidad del Quindío, Programa de Ingeniería Electrónica, 2012.

Vela, M.; Lindon, B. Teoria de juegos y modelos de oligopolio, 2011. Apuntes de estudio del curso de Economía de la Producción. Lambayeque - Peru. 